\title{
Remarks on Lorentz and CPT Violation in Field Theory
}

T. Mariz, J. R. Nascimento, and E. Passos

Departamento de Física, Universidade Federal da Paraíba, Caixa Postal 5008, 58051-970 João Pessoa, Paraíba, Brazil

Received on 10 August, 2006

\begin{abstract}
In this brief review we explicitly calculate the radiative corrections to the Chern-Simons-like term in the cases of zero and finite temperature, and in the gravity theory. Our results are obtained under the general guidance of dimensional regularization.
\end{abstract}

Keywords: Lorentz and CPT violation; Chern-Simons-like; Finite temperature

\section{INTRODUCTION}

The possibility of breaking Lorentz and CPT symmetries has been considered in several different contexts [1-6]. Most of them were dedicated to the extended quantum electrodynamics (QED) sector of the extended standard model (see e.g. Ref. [2, 3]). The initial motivation for consideration of Lorentz violation came from string theory $[7,8]$. Its basic idea is that interactions in the underlying theory induce nonzero expectation values for one or more Lorentz tensors which can be regarded as background quantities in the vacuum throughout spacetime.

Some time ago, in Ref. [1] the authors formulated the first model in which the electrodynamics is modified by adding the Chern-Simons-like term to Maxwell term and verified that the Lorentz and CPT symmetries are broken. This model predicts the rotation of the plane of polarization of light from distance galaxies, an effect which was not observed yet [1]. In recent papers $[9,10]$, the modification of general relativity obtained by adding the Chern-Simons-like gravitational term has been studied. The authors have observed that in such modified theory the Schwarzschild metric turns out to be a solution, gravitational waves possess two polarizations which travel with the velocity of light and polarized waves are suppressed or enhanced.

In four dimensions Colladay and Kostelecký posed the question whether such a term is induced when Lorentz and CPT violating term $\bar{\psi} b \gamma_{5} \psi$ is added to conventional Lagrangian of the QED [3]. In this case the fermions can be integrated out, and the radiative correction up to the first loop may lead to

$$
S_{C S}=\frac{1}{2} \int d^{4} x \varepsilon^{\mu \nu \lambda \rho} k_{\mu} F_{v \lambda} A_{\rho}
$$

where $k_{\mu}=C b_{\mu}$. The issue has been carefully investigated in several different contexts and regularization schemes, by many authors [3, 11-24], leading to results where $C$ vanishes $[3,5,17,18,24]$ and results where $C$ does not $[11-16,19-23]$ but different each other. This dependence upon the regularization scheme $[11,12]$ corresponds to an "ambiguity", i.e. finite but undetermined values, which has been well discussed in the literature $[25,26]$.

In this brief review we will summarize the induction of the Chern-Simons-like term by radiative corrections in the con- texts of the zero and finite temperature, and in the gravity theory.

\section{RADIATIVELY INDUCED CHERN-SIMONS-LIKE TERM}

In order to study this issue, one may consider the action of the Lorentz and CPT violating fermion sector

$$
S_{f}=\int d^{4} x \bar{\psi}\left(i \not \partial-m-\gamma_{5} \not b-\not\right) \psi \psi
$$

where $b_{\mu}$ is a constant four vector which selects a fixed direction in spacetime. To account for the fermionic integration we write

$$
e^{i S_{e f f}[b, A]}=\int D \bar{\psi} D \psi e^{i S_{f}}
$$

where the effective action is given by

$$
\left.S_{\text {eff }}[b, A]=-i \operatorname{Tr} \ln (i \not)-m-\gamma_{5} \not b-A\right) \psi \psi
$$

Note that the Eq. above can be written as $S_{\text {eff }}[b, A]=$ $S_{e f f}^{(0)}[b]+S_{e f f}^{(1)}[b, A]$, where $S_{\text {eff }}^{(0)}[b]=-i \operatorname{Tr} \ln \left(\not p-m-\gamma_{5} \not b\right)$ and

$$
S_{e f f}^{(1)}[b, A]=i \operatorname{Tr} \sum_{n=1}^{\infty} \frac{1}{n}\left[\frac{1}{\not p-m-\gamma_{5} \not \phi} A\right]^{n} .
$$

Since the term $S_{e f f}^{(0)}[b]$ is independent of the gauge field and cannot induce Chern-Simons-term, we shall focus only on the second term $S_{e f f}^{(1)}[b, A]$ looking for the first order derivative terms which are linear in $\not b$ and quadratic in $\not A$. This is the perturbative route, in which we do not include the contribution involving the vector $b_{\mu}$ into the Dirac propagator (see Ref. [11] for details).

Using the derivative expansion $[27,28]$

$$
\frac{1}{\not p-i \not \partial-m}=\frac{1}{\not p-m}+\frac{1}{\not p-m} i \not \partial \frac{1}{\not p-m}+\cdots,
$$

we can rewrite Eq.(5) in the form

$$
S_{e f f}^{(1 b)}[b, A]=\frac{i}{2} \int d^{4} x\left(\Pi_{1}^{\mu \nu}+\Pi_{2}^{\mu \nu}+\Pi_{3}^{\mu v}\right) A_{\mu} A_{v}
$$


where

$$
\begin{aligned}
& \Pi_{1}^{\mu \nu}=\operatorname{tr} \int \frac{d^{4} p}{(2 \pi)^{4}} S(p) b \gamma_{5} S(p) \gamma^{\mu} S(p) i \partial \partial S(p) \gamma^{v}, \\
& \Pi_{2}^{\mu \nu}=\operatorname{tr} \int \frac{d^{4} p}{(2 \pi)^{4}} S(p) \gamma^{\mu} S(p) b \gamma_{5} S(p) i \partial \partial S(p) \gamma^{v},
\end{aligned}
$$

and

$$
\Pi_{3}^{\mu \nu}=\operatorname{tr} \int \frac{d^{4} p}{(2 \pi)^{4}} S(p) \gamma^{\mu} S(p) i \not \partial S(p) b \gamma_{5} S(p) \gamma^{\nu}
$$

with $S(p)=(\not p-m)^{-1}$.

We evaluate the integrals under the general guidance of dimensional regularization [29-31]. Thus, we change dimensions from 4 to $2 w$, and we change $d^{4} p /(2 \pi)^{4}$ to $\left(\mu^{2}\right)^{2-w}\left[d^{2 w} p /(2 \pi)^{2 w}\right]$, where $\mu$ is an arbitrary parameter that identifies the mass scale. We use two distinct routes to do the calculations involving the Dirac matrices. In the first route we use the cyclic property of the trace, to move $\gamma_{5}$ to the very end of every expression involving the trace of Dirac matrices and $\gamma^{\alpha} \gamma_{\alpha}=2 w$, so that we get

$$
\begin{aligned}
S_{e f f}^{(1 b)}[b, A]= & \frac{3}{2} i \Pi(w) \operatorname{tr}\left(\gamma^{\mu} \gamma^{v} \gamma^{\lambda} \gamma^{\rho} \gamma_{5}\right) \\
& \times b_{\mu} \int d^{4} x \partial_{v} A_{\lambda} A_{\rho} .
\end{aligned}
$$

Here the factor 3 accounts for identical contributions that comes from $\Pi_{1}^{\mu \nu}, \Pi_{2}^{\mu \nu}$ and $\Pi_{3}^{\mu \nu}$, and $\Pi(w)$ is given by

$$
\begin{aligned}
\Pi(w)= & -\frac{2 w-1}{96 \pi^{2}}+\frac{w+1}{96 \pi^{2}}\left(\frac{4 \pi \mu^{2}}{m^{2}}\right)^{2-w} \\
& \times \Gamma(2-w)(2-w) .
\end{aligned}
$$

In the above calculations we have set $\Pi_{i}^{\mu \nu}=\Pi_{i, \operatorname{div}}^{\mu \nu}+\Pi_{i, \text { fin }}^{\mu \nu}$ to split the $\Pi_{i}^{\mu v}$ contribution into two parts, one divergent and the other finite. The contribution $\Pi_{i, \text { div }}^{\mu v}$ is divergent in the limit $w \rightarrow 2$, and it contribute with the term proportional to $\Gamma(2-w)$. However, the factor involving the Dirac matrices contributes with the term $(2-w)$, in a way such that the full contribution is finite in the limit $w \rightarrow 2$. Furthermore, this finite term exactly compensates the finite contribution that appears from $\Pi_{i \text {,fin }}^{\mu \nu}$ in the limit $w \rightarrow 2$. In the limit $w \rightarrow 2$ we can use $\operatorname{tr}\left(\gamma^{\mu} \gamma^{\nu} \gamma^{\lambda} \gamma^{\rho} \gamma_{5}\right)=4 i \varepsilon^{\mu \nu \lambda \rho}$, but $\Pi(w \rightarrow 2) \rightarrow 0$ and this leaves no room for Lorentz and CPT violation. The perfect balance between the two contributions that we have just found has been identified before in Ref. [34] as being peculiar to dimensional regularization.

We stress that if one uses the relation $\left\{\gamma^{\mu}, \gamma_{5}\right\}=0$ to move $\gamma_{5}$ to the end of every expression involving the trace of Dirac matrices, the perfect balance between the two contributions is broken, giving rise to a non zero value for the constant $C$. In the same way, if one uses the cyclic property of the trace and $\gamma^{\alpha} \gamma_{\alpha}=4$ [13], we have

$$
k_{\mu}=\frac{3}{16 \pi^{2}} b_{\mu}
$$

This is the unambiguous Chern-Simons coefficient [11] obtained when we use the nonperturbative route, in which we include the contribution involving $\not b$ into the Dirac propagator.

We make this point stronger by considering another route to implement the calculation involving properties of the Dirac matrices when the spacetime has dimension $2 w$. We follow $[31,32]$, and now the Dirac matrices contracted with $\not b$ and $\not A$ are physical matrices; they are written in the form $\bar{\gamma}^{\mu}$, etc. The other Dirac matrices are changed according to the rule $\gamma^{\alpha} \rightarrow \bar{\gamma}^{\alpha}+\hat{\gamma}^{\alpha}$, where $\left\{\bar{\gamma}^{\alpha}, \bar{\gamma}^{\beta}\right\}=2 \bar{g}^{\alpha \beta},\left\{\hat{\gamma}^{\alpha}, \hat{\gamma}^{\beta}\right\}=2 \hat{g}^{\alpha \beta}$, and $\left\{\bar{\gamma}^{\alpha}, \hat{\gamma}^{\beta}\right\}=0$, and also $\bar{\gamma}^{\alpha} \bar{\gamma}_{\alpha}=4, \bar{\gamma}^{\alpha} \hat{\gamma}_{\alpha}=0$ and $\hat{\gamma}^{\alpha} \hat{\gamma}_{\alpha}=2(w-2)$. In this case we can use either the cyclic property of the trace, or the relations $\left\{\gamma_{5}, \bar{\gamma}^{\mu}\right\}=\left[\gamma_{5}, \hat{\gamma}^{\mu}\right]=0$ with $\operatorname{tr}\left(\bar{\gamma}^{\mu} \bar{\gamma}^{v} \bar{\gamma}^{\lambda} \bar{\gamma}^{\rho} \gamma_{5}\right)=$ $4 i \varepsilon^{\mu \nu \lambda \rho}$ and $\operatorname{tr}\left(\gamma^{\mu} \gamma^{v} \gamma^{\lambda} \hat{\gamma}^{\rho} \gamma_{5}\right)=0$, that we arrive at the same result where the Eq. (12) does vanish.

Therefore, we can emphasize that concerning dimensional regularization scheme a variety of results can be obtained when a variety of prescriptions is made.

\section{RADIATIVELY INDUCED CHERN-SIMONS-LIKE TERM AT FINITE TEMPERATURE}

In this section we will analyze the behavior of the parameter $\mathrm{C}$ when we take temperature into account. By comparing with results in the literature [33-35], we also will find that at finite temperature, the Chern-Simons-like term remains undetermined. It is more convenient rewrite the Eq. (4) as $S_{\text {eff }}[b, A]=S_{\text {eff }}^{(0)}[b]+S_{\text {eff }}^{(1)}[b, A]$, where now

$$
S_{e f f}^{(1)}[b, A]=i \int_{0}^{1} d z \operatorname{Tr}\left[\frac{1}{i \partial \gamma^{\prime}-m-\gamma_{5} \not b-z A(x)} A(x)\right] .
$$

To perform the momentum space integration in Eq. (14), we consider the prescription [37]:

$$
i \partial{ }^{\prime} \rightarrow \not p, \quad \quad A(x) \rightarrow A d\left(x-i \frac{\partial}{\partial p}\right) .
$$


Then, the Eq. (14) now reads

$$
S_{e f f}^{(1)}[b, A]=i \int_{0}^{1} d z \int d^{4} x \int \frac{d^{4} p}{(2 \pi)^{4}} \operatorname{tr}\left[\frac{1}{\not p-m-\gamma_{5} \not b-z e A\left(x-i \frac{\partial}{\partial p}\right)} A(x)\right] .
$$

We can manipulate the Eq.(16) to keep only first order derivative terms which are linear in $\not b$ and quadratic in $\not A$. Carrying out the integral in $z$ gives

$$
S_{e f f}^{(1 b)}[b, A]=-\frac{i}{2} \int d^{4} x \int \frac{d^{4} p}{(2 \pi)^{4}} \operatorname{tr}\left[\frac{1}{\not p-m} i \partial_{\mu} A \frac{\partial}{\partial p_{\mu}} \frac{1}{\not p-m} \gamma_{5} \not \phi \frac{1}{\not p-m} \not A+\frac{1}{\not p-m} \gamma_{5} \not b \frac{1}{\not p-m} i \partial_{\mu} A \frac{\partial}{\partial p_{\mu}} \frac{1}{\not p-m} A\right] .
$$

Now, using the relation

$$
\frac{\partial}{\partial p_{\mu}} \frac{1}{\not p-m}=-\frac{1}{\not p-m} \gamma^{\mu} \frac{1}{\not p-m} .
$$

and taking the traces of the products of $\gamma$ matrices on relevant terms, i.e., the terms that contain $\operatorname{tr}\left(\gamma^{\mu} \gamma^{v} \gamma^{\alpha} \gamma^{\beta} \gamma_{5}\right)$, the Eq.(17) takes the form

$$
S_{e f f}^{(1 b)}[b, A]=-\frac{1}{2} \int d^{4} x \int \frac{d^{4} p}{(2 \pi)^{4}} \frac{N}{\left(p^{2}-m^{2}\right)^{4}},
$$

where $N$ is given by

$$
\begin{aligned}
N & =-4 i\left(p^{2}-m^{2}\right)\left[\varepsilon^{\alpha \beta \mu \sigma}\left(3 m^{2}+p^{2}\right)-4 \varepsilon^{\alpha \beta \mu \nu} p_{v} p^{\sigma}\right] \\
& \times b_{\sigma} \partial_{\mu} A_{\alpha} A_{\beta} .
\end{aligned}
$$

Note that by power counting the momentum integral in Eq. (18) contains terms with logarithmic divergence. Let us use the relation

$$
\int \frac{d^{D} q}{(2 \pi)^{D}} q_{\mu} q_{v} f\left(q^{2}\right)=\frac{g_{\mu \nu}}{D} \int \frac{d^{D} q}{(2 \pi)^{D}} q^{2} f\left(q^{2}\right),
$$

that naturally removes the logarithmic divergence. Now, considering $D=4$, the terms containing $p^{2}$ and $p_{v} p^{\sigma}$ in (19) cancel out and we find

$$
N=-12 m^{2} i\left(p^{2}-m^{2}\right) \varepsilon^{\alpha \beta \mu \sigma} b_{\sigma} \partial_{\mu} A_{\alpha} A_{\beta}
$$

In this way, the logarithmic divergence in (18) disappears, so that the effective action now reads

$$
\begin{aligned}
S_{e f f}^{(1 b)}[b, A] & =\left[6 \operatorname{im}^{2} \int \frac{d^{4} p}{(2 \pi)^{4}} \frac{1}{\left(p^{2}-m^{2}\right)^{3}}\right] \\
& \times \varepsilon^{\alpha \beta \mu \sigma} b_{\sigma} \int d^{4} x \partial_{\mu} A_{\alpha} A_{\beta},
\end{aligned}
$$

which is finite by power counting. Evaluating the momentum integral in the (22) we also obtain unambiguously the ChernSimons coefficient [11]

$$
k_{\mu}=\frac{3}{16 \pi^{2}} b_{\mu}
$$

However, if we use another regularization scheme $k_{\mu}$ may vanish, for instance, in Pauli-Villars regularization scheme [3].
Next we study such undetermined coefficient when we take into account the temperature.

Let us now assume that the system is at thermal equilibrium with a temperature $T=1 / \beta$. In this case we can use Matsubara formalism for fermions, which consists in taking $p_{0}=(n+1 / 2) 2 \pi / \beta$ and changing $(1 / 2 \pi) \int d p_{0}=1 / \beta \sum_{n}$ [38]. We also change the Minkowski space to Euclidean space, by making $x_{0}=-i x_{4}, p_{0}=i p_{4}$ and $b_{0}=i b_{4}$, such that $p^{2}=-p_{E}^{2}, p_{E}^{2}=\mathbf{p}^{2}+p_{4}^{2}, d^{4} p=i d^{4} p_{E}$ and $d^{4} x=-i d^{4} x_{E}$. Now the Eq. (22) can be written as

$$
S_{e f f}^{(1 b)}[b, A]=6 f\left(m^{2}, \beta\right) \varepsilon^{\alpha \beta \mu \sigma} b_{\sigma} \int(-i) d^{4} x_{E} \partial_{\mu} A_{\alpha} A_{\beta},
$$

where $f\left(m^{2}, \beta\right)$, is the Chern-Simons coefficient dependent on the temperature which is given by

$$
\begin{aligned}
f\left(m^{2}, \beta\right) & =\frac{m^{2}}{\beta} \int \frac{d^{3} \mathbf{p}}{(2 \pi)^{3}} \sum_{n=-\infty}^{\infty} \frac{1}{\left(\mathbf{p}^{2}+p_{4}^{2}+m^{2}\right)^{3}} \\
& =\frac{m^{2}}{2 \beta} \frac{d^{2}}{d\left(m^{2}\right)^{2}} \int \frac{d^{3} \mathbf{p}}{(2 \pi)^{3}} \sum_{n=-\infty}^{\infty} \frac{1}{\left(\mathbf{p}^{2}+p_{4}^{2}+m^{2}\right)^{2}}
\end{aligned}
$$

We calculate the momentum integral by adopting dimensional regularization scheme to obtain

$$
f\left(m^{2}, \beta\right)=\frac{m^{2}}{2 \beta} \frac{\Gamma(3-D / 2)}{(4 \pi)^{D / 2}} \sum_{n=-\infty}^{\infty} \frac{1}{\left(p_{4}^{2}+m^{2}\right)^{3-D / 2}} .
$$

To perform summation we shall use an explicit representation for the sum over the Matsubara frequencies [39]:

$$
\begin{gathered}
\sum_{n}\left[(n+b)^{2}+a^{2}\right]^{-\lambda}=\frac{\sqrt{\pi} \Gamma(\lambda-1 / 2)}{\Gamma(\lambda)\left(a^{2}\right)^{\lambda-1 / 2}} \\
+4 \sin (\pi \lambda) \int_{|a|}^{\infty} \frac{d z}{\left(z^{2}-a^{2}\right)^{\lambda}} \operatorname{Re}\left(\frac{1}{\exp 2 \pi(z+i b)-1}\right),
\end{gathered}
$$

which is valid for $1 / 2<\lambda<1$. This implies that for $\lambda=$ $3-D / 2$ as given in Eq.(26) we cannot apply this relation for $D=3$, because the integral in (27) does not converge. Thus, let us perform the analytic continuation of this relation, so we obtain 


$$
\begin{aligned}
\int_{|a|}^{\infty} \frac{d z}{\left(z^{2}-a^{2}\right)^{\lambda}} \operatorname{Re}\left(\frac{1}{\exp 2 \pi(z+i b)-1}\right)= & \frac{1}{2 a^{2}} \frac{3-2 \lambda}{1-\lambda} \int_{|a|}^{\infty} \frac{d z}{\left(z^{2}-a^{2}\right)^{\lambda-1}} \operatorname{Re}\left(\frac{1}{\exp 2 \pi(z+i b)-1}\right) \\
& -\frac{1}{4 a^{2}} \frac{1}{(2-\lambda)(1-\lambda)} \int_{|a|}^{\infty} \frac{d z}{\left(z^{2}-a^{2}\right)^{\lambda-2}} \frac{d^{2}}{d z^{2}} \operatorname{Re}\left(\frac{1}{\exp 2 \pi(z+i b)-1}\right) .
\end{aligned}
$$

Now for $D=3$ the Eq.(26) takes the form [36]

$$
f\left(m^{2}, \beta\right)=\frac{1}{32 \pi^{2}}+\frac{1}{16} F(\xi),
$$

where $\xi=\frac{\beta m}{2 \pi}$ and the function

$$
F(\xi)=\int_{|\xi|}^{\infty} d z\left(z^{2}-\xi^{2}\right)^{1 / 2} \frac{\tanh (\pi z)}{\cosh ^{2}(\pi z)},
$$

approaches the limits: $F(\xi \rightarrow \infty) \rightarrow 0(T \rightarrow 0)$ and $F(\xi \rightarrow$ $0) \rightarrow 1 / 2 \pi^{2}(T \rightarrow \infty)$ - see Fig.1. Thus, we see that at high temperature the Chern-Simons coefficient is twice its value at zero temperature, i.e., $f\left(m^{2}, \beta \rightarrow 0\right)=1 / 16 \pi^{2}$. On the other hand, at zero temperature, one recovers the result (23).

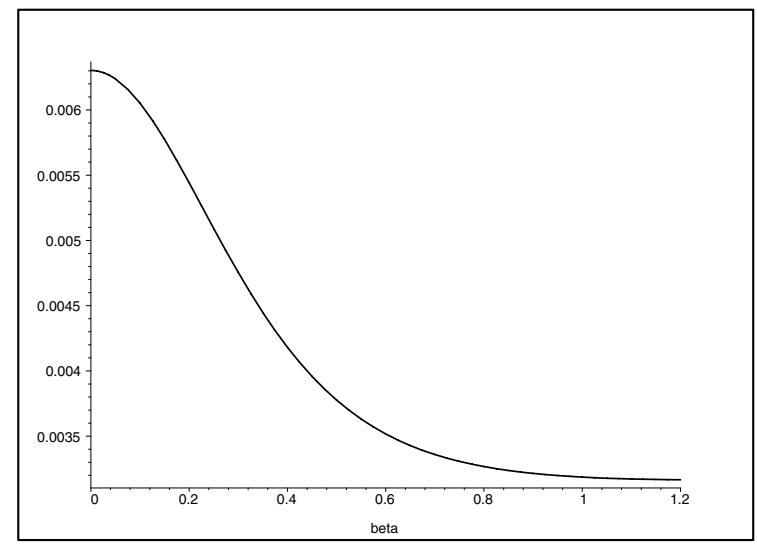

FIG. 1: The function $f\left(m^{2}, \beta\right)$ is different from zero everywhere. At zero temperature $(\beta \rightarrow \infty)$, the function tends to a nonzero value $1 / 32 \pi^{2}$.

\section{RADIATIVELY INDUCED CHERN-SIMONS-LIKE TERM IN GENERAL RELATIVITY}

The action that we are interested is given by

$$
S=\int \mathrm{d}^{4} x\left(\frac{1}{2} i e e^{\mu}{ }_{a} \bar{\psi} \gamma^{a} \stackrel{\leftrightarrow}{D}_{\mu} \psi-e e^{\mu}{ }_{a} \bar{\psi} b_{\mu} \gamma^{a} \gamma_{5} \psi\right)
$$

where we have included the parity-violating term. Here, $e_{a}^{\mu}$ is the tetrad (vierbein), $e \equiv \operatorname{det} e^{\mu}{ }_{a}$ and $b_{\mu}$ is a constant 4 -vector. The covariant derivative is given by

$$
D_{\mu} \psi=\partial_{\mu} \psi+\frac{1}{2} w_{\mu c d} \sigma^{c d} \psi
$$

where $w_{\mu}^{c d}$ is the spin connection and $\sigma^{c d}=\frac{1}{4}\left[\gamma^{c}, \gamma^{d}\right]$, whereas the covariant derivative on a Dirac-conjugate field $\bar{\psi}$ is

$$
D_{\mu} \bar{\psi}=\partial_{\mu} \bar{\psi}-\frac{1}{2} w_{\mu c d} \bar{\psi} \sigma^{c d} \text {. }
$$

Using the expressions above we can rewrite the Eq. (31) as follow

$$
\begin{aligned}
S= & \int \mathrm{d}^{4} x\left(\frac{1}{2} i e e^{\mu}{ }_{a} \bar{\psi} \gamma^{a} \overleftrightarrow{\partial}_{\mu} \psi+\frac{1}{4} i e e_{a}^{\mu} \bar{\psi} w_{\mu c d} \Gamma^{a c d} \psi\right. \\
& \left.-e e_{a}^{\mu} \bar{\psi} b_{\mu} \gamma^{a} \gamma_{5} \psi\right),
\end{aligned}
$$

where $\Gamma^{a c d}=\frac{1}{6}\left(\gamma^{a} \gamma^{c} \gamma^{d} \pm\right.$ permutations $)$, i.e. the antisymmetrized product of three $\gamma$-matrices.

In the weak field approximation we consider $g_{\mu v}=\eta_{\mu v}+$ $h_{\mu v}\left(g^{\mu \nu}=\eta^{\mu \nu}-h^{\mu v}\right)$, which induces an expansion for the vierbein $e_{\mu a}=\eta_{\mu a}+\frac{1}{2} h_{\mu a}\left(e_{a}^{\mu}=\eta_{a}^{\mu}-\frac{1}{2} h_{a}^{\mu}\right)$. Then, the linearized Chern-Simons-like action takes the form [9]

$$
S_{\text {linear }}=\frac{1}{4} \int d^{4} x h^{\mu \nu} v^{\lambda} \varepsilon_{\alpha \mu \lambda \rho} \partial^{\rho}\left(\partial_{\gamma} \partial^{\gamma} h_{v}^{\alpha}-\partial_{v} \partial_{\gamma} h^{\gamma \alpha}\right) .
$$

The main goal here is to induce this action by radiative correction of fermionic matter field obtaining the relation between $v_{\lambda}$ and $b_{\mu}$ [40]. In order to perform this calculation we consider the fermionic model represented by the action

$$
e^{i \Gamma[h]}=\int \mathcal{D} \bar{\psi} \mathcal{D} \psi e^{i S[h, \bar{\psi}, \psi]},
$$

where the linearized effective action is given by

$$
S[h, \bar{\psi}, \psi]=\int \mathrm{d}^{4} x\left(\frac{1}{2} i \bar{\psi} \Gamma^{\mu} \overleftrightarrow{\partial}_{\mu} \psi+\bar{\psi} h_{\mu v} \Gamma^{\mu v} \psi-\bar{\psi} b_{\mu} \gamma^{\mu} \gamma_{5} \psi\right),
$$

with $\Gamma^{\mu}=\gamma^{\mu}-\frac{1}{2} h^{\mu v} \gamma_{v}$ and $\Gamma^{\mu v}=\frac{1}{2} b^{\mu} \gamma^{v} \gamma_{5}-$ $\frac{i}{16}\left(\partial_{\rho} h_{\alpha \beta}\right) \eta^{\beta v} \Gamma^{\rho \mu \alpha}$. In this expression, we neglect the terms proportional to $h=\eta^{\mu v} h_{\mu \nu}$ because they do not contribute to generating of the Chern-Simons-like action.

The Feynman rules that we obtain from Eq.(37) are:

$$
\begin{aligned}
& -\quad=S(p)=\frac{i}{\not p-m} \\
& \sim \quad=-i \not \gamma \gamma_{5} \\
& \begin{array}{ll} 
& =-\frac{i}{4} \gamma_{\mu}(2 p+q)_{v} \\
i & =i \gamma_{\mu} b_{v} \gamma_{5} \\
\}_{j} & =-\frac{i}{16} \eta^{\beta v} \Gamma^{\mu \rho \alpha}\left(q_{1}-q_{2}\right)_{\rho} .
\end{array}
\end{aligned}
$$

The relevant one-loop graphs to the Chern-Simons-like effective action are shown in the Fig. (2), whose Feynman integrals are given by 


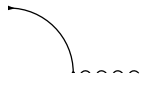

(a)

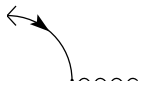

(b)

FIG. 2: One-loop relevant contributions

$$
\Pi_{a}^{\mu \nu \alpha \beta}(q)=-\frac{i}{16} \operatorname{tr} \int \frac{\mathrm{d}^{4} p}{(2 \pi)^{4}}\left[\gamma^{\mu}(2 p+q)^{v} S(p) \gamma^{\alpha}(2 p+q)^{\beta} S(p+q) \not \gamma \gamma_{5} S(p+q)\right]
$$

and

$$
\Pi_{b}^{\mu \nu \alpha \beta}(q)=-\frac{i}{16} \operatorname{tr} \int \frac{\mathrm{d}^{4} p}{(2 \pi)^{4}}\left[\gamma^{\mu}(2 p+q)^{v} S(p) \not b \gamma_{5} S(p) \gamma^{\alpha}(2 p+q)^{\beta} S(p+q)\right] .
$$

It is straightforward to see that

$$
\Pi^{\mu v \alpha \beta}(q)=\Pi_{a}^{\mu \nu \alpha \beta}(q)=\Pi_{b}^{\alpha v \mu \beta}(-q),
$$

which appears of substituting the loop momenta, $p \rightarrow p-q x$, and we use the cyclic properties of the trace of a product of $\gamma$-matrices. So, from now on we work only with Eq. (39) which takes the form

$$
\begin{aligned}
\Pi^{\mu v \alpha \beta}(q)= & -\frac{1}{8} \int_{0}^{1} \mathrm{~d} x x \int \frac{\mathrm{d}^{4} p}{(2 \pi)^{4}} \frac{(2 p+q(1-2 x))^{v}(2 p+q(1-2 x))^{\beta}}{\left[p^{2}-m^{2}+x(1-x) q^{2}\right]^{3}} \\
& \times \operatorname{tr}\left[\gamma^{\mu}(\not p-\not q x+m) \gamma^{\alpha}(\not p+\not q(1-x)+m) \not b \gamma_{5}(\not p+\not q(1-x)+m)\right],
\end{aligned}
$$

where we have used Feynman parameter to combine the denominator in Eq. (42). Taking into account the trace of Dirac matrices and dropping all odd terms in $p$, we get

$$
\Pi^{\mu \nu \alpha \beta}(q)=-\frac{1}{8} \int_{0}^{1} d x x \int \frac{d^{4} p}{(2 \pi)^{4}} \frac{N^{\mu \nu \alpha \beta}\left(p^{0}, p^{2}, p^{4}\right)}{\left[p^{2}-m^{2}+x(1-x) q^{2}\right]^{3}},
$$

where the numerator $N^{\mu v \alpha \beta}\left(p^{0}, p^{2}, p^{4}\right)$ has the form,

$$
N^{\mu \nu \alpha \beta}\left(p^{0}, p^{2}, p^{4}\right)=4 p^{v} p^{\beta}\left(T_{0}^{\alpha \mu}+T_{p p}^{\alpha \mu}\right)+2(1-2 x)\left(p^{v} q^{\beta}+p^{\beta} q^{v}\right)\left(T_{p}^{\alpha \mu}+T_{p p p}^{\alpha \mu}\right)+(1-2 x)^{2} q^{v} q^{\beta}\left(T_{0}^{\alpha \mu}+T_{p p}^{\alpha \mu}\right)
$$

with

$$
\begin{aligned}
T_{0}^{\alpha \mu} & =-4 i b_{\lambda} \varepsilon^{\alpha \mu \lambda \theta} q_{\theta}\left[x(1-x)^{2} q^{2}+(2-x) m^{2}\right], \\
T_{p}^{\alpha \mu} & =-4 i b_{\lambda} \varepsilon^{\alpha \mu \lambda \rho}\left[m^{2}+\left(1-x^{2}\right) q^{2}\right] p_{\rho}-8 i(1-x) b_{\lambda}\left[\varepsilon^{\mu \lambda \rho \theta} q^{\alpha}-\varepsilon^{\alpha \lambda \rho \theta} q^{\mu}-(1-x) \varepsilon^{\alpha \mu \lambda \theta} q^{\rho}\right] q_{\theta} p_{\rho}, \\
T_{p p}^{\alpha \mu} & =-4 i b_{\lambda}\left[2\left(\varepsilon^{\mu \lambda \rho \theta} p_{\rho} p^{\alpha}-\varepsilon^{\alpha \lambda \rho \theta} p_{\rho} p^{\mu}+x \varepsilon^{\alpha \mu \lambda \rho} p_{\rho} p^{\theta}\right)-(2-x) \varepsilon^{\alpha \mu \lambda \theta} p^{2}\right] q_{\theta}, \\
T_{p p p}^{\alpha \mu} & =4 i b_{\lambda} \varepsilon^{\alpha \mu \lambda \rho} p^{2} p_{\rho} .
\end{aligned}
$$

The integral (43) is badly divergent. Finally, using the dimensional regularization, the Eq. (42) takes the form

$$
\Pi^{\mu \nu \alpha \beta}(q)=b_{\lambda} \varepsilon^{\alpha \mu \lambda \rho} q_{\rho}\left[A q^{2} \eta^{\beta v}+B q^{\beta} q^{v}\right]
$$

where the expressions $A$ and $B$ are given by

$$
\begin{aligned}
A= & \frac{1}{32 \pi^{2}} \int_{0}^{1} \mathrm{~d} x\left[3 x^{3}(1-x)+(5 x-3) x^{2}(1-x)\left(\frac{2}{\varepsilon}+\ln \left(\frac{4 \pi \mu^{2}}{-M^{2}}\right)-\gamma\right)\right. \\
& \left.-3 x^{2}\left(\frac{2}{\varepsilon}+\ln \left(\frac{4 \pi \mu^{2}}{-M^{2}}\right)-\gamma+1\right) \frac{m^{2}}{q^{2}}\right]
\end{aligned}
$$


and

$$
\begin{aligned}
B= & \frac{1}{64 \pi^{2}} \int_{0}^{1} \mathrm{~d} x\left\{(1-2 x)^{2}(3-2 x) \frac{x^{2}(1-x) q^{2}}{M^{2}}\right. \\
& \left.+[(2-3 x)(1-2 x)-4 x(1-x)] x(1-2 x)\left(\frac{2}{\varepsilon}+\ln \left(\frac{4 \pi \mu^{2}}{-M^{2}}\right)-\gamma\right)\right\},
\end{aligned}
$$

after they have been expanded around $\varepsilon \rightarrow 0$, with $\varepsilon=4-D$ and $M^{2}=m^{2}-x(1-x) q^{2}$.

As one can see $\int_{0}^{1} d x\left[(5 x-3) x^{2}(1-x)\right]\left(\frac{2}{\varepsilon}-\gamma\right)=0$ in $A$ and $\int_{0}^{1} d x[(2-3 x)(1-2 x)-4 x(1-x)] x(1-2 x)\left(\frac{2}{\varepsilon}-\gamma\right)=0$ in $B$, then $A$ and $B$ take the form

$$
\begin{aligned}
A & =\frac{1}{32 \pi^{2}} \int_{0}^{1} \mathrm{~d} x\left[3 x^{3}(1-x)+\frac{(1-2 x) x^{3}(1-x)^{2} q^{2}}{m^{2}-x(1-x) q^{2}}\right. \\
& \left.-3 x^{2}\left(\frac{2}{\varepsilon}+\ln \left(\frac{4 \pi \mu^{2}}{-M^{2}}\right)-\gamma+1\right) \frac{m^{2}}{q^{2}}\right]
\end{aligned}
$$

and

$$
B=\frac{1}{32 \pi^{2}} \int_{0}^{1} \mathrm{~d} x \frac{(1-2 x)^{2} x^{2}(1-x) q^{2}}{m^{2}-x(1-x) q^{2}} .
$$

Here we have performed an integration by parts on $x$ for log term in $A$ and $B$. Note that in $A$ the divergent part is present which will disappear when we consider the limit $m^{2} \rightarrow 0$. Now performing the $x$-integration, we have

$$
\left.A\right|_{m^{2} \rightarrow 0}=-\left.B\right|_{m^{2} \rightarrow 0}=\frac{1}{192 \pi^{2}} .
$$

We substitute these results into Eq. (49), to obtain the ChernSimons-like term

$$
\Pi^{\mu v \alpha \beta}(q)=\frac{1}{192 \pi^{2}} b_{\lambda} \varepsilon^{\alpha \mu \lambda \rho} q_{\rho}\left[q^{2} \eta^{\beta v}-q^{\beta} q^{v}\right] .
$$

Finally, the Chern-Simons-like gravitational action induced by radiatively corrections is given by

$$
\Gamma_{\mathrm{cs}}[h]=\frac{1}{192 \pi^{2}} \int \mathrm{d}^{4} x b^{\lambda} h^{\mu v} \varepsilon_{\alpha \mu \lambda \rho} \partial^{\rho}\left[\partial_{\gamma} \partial^{\gamma} h_{v}^{\alpha}-\partial_{v} \partial_{\gamma} h^{\gamma \alpha}\right] .
$$

Comparing to Eq.(35) we obtain the relation between the parameters $v_{\lambda}$ and $b_{\mu}$ which is written as

$$
v_{\lambda}=\frac{1}{48 \pi^{2}} b_{\lambda}
$$

\section{CONCLUSION}

We have studied the induction of Chern-Simons-like term at zero and finite temperature, and in gravity theory. Here the dimensional regularization was applied to evaluate momentum integrals. We found that depending on the prescription, one can obtain either zero Chern-Simons coefficient or the unambiguous result [11], at zero temperature.

At finite temperature, our result is finite [36] but does not agree with other results presented in the literature [33-35]. We argue that this is also caused by different regularization schemes. In the limit $T \rightarrow 0$ our result leads to a nonzero Chern-Simons-like term, a behavior also predicted in [33], obtained with the use of dimensional regularization, and the result in [35], obtained with the use of cutoff regularization scheme. However, it is in conflict with the result found in [34] which suggests the vanishing of the Chern-Simons-like term at zero temperature. On the other hand, at high temperature our result behaves as the result of [34]. But now, however, it conflicts with the results in $[33,35]$ which predict that the Chern-Simons-like term vanishes at high temperature. These results are all finite, and they show that the Chern-Simons coefficient is indeed undetermined just as it happens at zero temperature $[25,26]$.

Finally, we recall that we also have calculated the radiative corrections induced by Dirac fermions coupled to a gravitational background field, including the nonstandard contribution that violates parity. In this calculation we have used the weak field approximation. As we are using the perturbative route, i.e., to lowest order in $b_{\mu}$, probably these radiative corrections are also undetermined.

\section{Acknowledgments}

This work was done in collaboration with D. Bazeia, R. F. Ribeiro and F. A. Brito. We would like to thank to A. Yu. Petrov for comments and discussions, and CAPES, CNPq, PADCT/CNPq, and PRONEX/CNPq/FAPESQ for financial support.
[1] S. Carroll, G. Field, and R. Jackiw, Phys. Rev. D 41, 1231 (1990).

[2] D. Colladay and V. A. Kostelecký, Phys. Rev. D 55, 6760 (1997).

[3] D. Colladay and V. A. Kostelecký, Phys. Rev. D 58, 116002, (1998)
[4] S. Coleman and S. L. Glashow, Phys. Lett. 405B, 249 (1997).

[5] S. Coleman and S. L. Glashow, Phys. Rev. D 59, 116008, (1999).

[6] V. A. Kostelecky, ed., CPT and Lorentz Symmetry, World Scientific, Singapore, 1999

[7] V. A. Kostelecký and S. Samuel. Phys. Rev. D 39683 (1989) 
[8] V. A. Kostelecký and S. Samuel Phys. Rev. Lett. 63224 (1989).

[9] R. Jackiw and S.-Y. Pi, Phys. Rev. D 68104012 (2003).

[10] A. Lue, L. Wang, and M. Kamionkowski, Phys. Rev. Lett. 83, 1506 (1999).

[11] R. Jackiw and V.A. Kostelecký, Phys. Rev. Lett. 82, 3572 (1999).

[12] M. Pérez-Vitoria, Phys. Rev. Lett. 83, 2518 (1999).

[13] J. M. Chung and P. Oh, Phys. Rev. D 60, 067702 (1999).

[14] J. M. Chung, Phys. Rev. D 60, 127901 (1999).

[15] W. F. Chen, Phys. Rev. D 60, 085007 (1999).

[16] J. M. Chung, Phys. Lett. B 461, 318 (1999).

[17] C. Adam and F. R. Klinkhamer, Nuc. Phys. B 607, 247 (2001); Phys. Lett. B 513, 245 (2001).

[18] G. Bonneau, Nucl. Phys. B 593, 398 (2001).

[19] Yu. A. Sitenko, Phys. Lett. B 515, 414 (2001).

[20] M. Pérez-Victoria, J. High Energy Phys. 04, 032 (2001).

[21] M. Chaichian, W.F. Chen, and R. González Felipe, Phys. Lett. B 503, 215 (2001).

[22] J. M. Chung and B.K. Chung, Phys. Rev. D 63, 105015 (2001).

[23] A. A. Andrianov, P. Giacconi, and R. Soldati, J. High Energy Phys. 02, 030 (2002).

[24] D. Bazeia, T. Mariz, J. R. Nascimento, E. Passos, and R. F. Ribeiro, J. Phys. A 364937 (2003)

[25] R. Jackiw, Int. J. Mod. Phys. B14, 2011 (2000).

[26] M. Perez-Victoria, JHEP 0104, 032 (2001).
[27] I. J. R. Aitchison and C. M. Fraser, Phys. Lett. B 146, 63 (1984); Phys. Rev. D 31, 2605 (1985); C. M. Fraser, Z. Phys. C 28, 101 (1985).

[28] A. Das and A. Karev, Phys. Rev. D 36, 623 (1987); K. S. Babu, A. Das, and P. Panagrahi, Phys. Rev. D 36, 3725 (1987).

[29] J. Ashmore, Lett. Nuovo Cim. 4, 289 (1972).

[30] C. G. Bolini and J. J. Giambiagi, Phys. Lett. B 40, 566 (1972).

[31] G. t'Hooft and M. J. G. Veltman, Nucl. Phys. B 44, 189 (1972).

[32] P. Breitenlohner and D. Maison, Comm. Math. Phys. 52, 11 (1977).

[33] J. R. Nascimento, R. F. Ribeiro, and N. F. Svaiter, Radiatively induced Lorentz and CPT violation in QED at finite temperature, hep-th/0012039.

[34] L. Cervi, L. Griguolo, and D. Seminara, Phys. Rev. D 64, 105003 (2001).

[35] D. Ebert, V. C. Zhukovsky, and A. S. Razumovsky, Phys. Rev. D 70, 025003 (2004).

[36] T. Mariz, J. R. Nascimento, E. Passos, R. F. Ribeiro, and F. A. Brito, J. High Energy Phys. 10, 019 (2005).

[37] L. H. Chan, Phys. Rev. Lett, 54, 1222 (1985).

[38] L. Dolan and R. Jackiw, Phys. Rev. D 9, 3320 (1974).

[39] L. H. Ford, Phys. Rev. D 21, 933 (1980).

[40] T. Mariz, J. R. Nascimento, E. Passos, and R. F. Ribeiro, Phys. Rev. D 70, 024014 (2004). 